\title{
The CMUnited-98 Champion Simulator Team *
}

\author{
Peter Stone, Manuela Veloso, and Patrick Riley \\ Computer Science Department, Carnegie Mellon University \\ Pittsburgh, PA 15213 \\ \{pstone,veloso\}@cs.cmu.edu, priley@andrew.cmu.edu
}

\begin{abstract}
The CMUnited-98 simulator team became the 1998 RoboCup simulator league champion by winning all 8 of its games, outscoring opponents by a total of $66-0$. CMUnited-98 builds upon the successful CMUnited-97 implementation, but also improves upon it in many ways. This chapter describes the complete CMUnited-98 software, emphasizing the recent improvements. Coupled with the publicly-available CMUnited-98 source code, it is designed to help other RoboCup and multi-agent systems researchers build upon our success.
\end{abstract}

\section{Introduction}

The CMUnited-98 simulator team became the 1998 RoboCup [4] simulator league champion by winning all 8 of its games, outscoring opponents by a total of $66-0$. CMUnited-98 builds upon the successful CMUnited-97 implementation [8], but also improves upon it in many ways.

The most notable improvements are the individual agent skills and the strategic agent positioning in anticipation of passes from teammates. While the success of CMUnited-98 also depended on our previous research innovations including layered learning [9], a flexible teamwork structure [10], and a novel communication paradigm [10], these techniques are all described elsewhere. The purpose of this article is to clearly and fully describe the low-level CMUnited-98 agent architecture as well as the key improvements over the previous implementation.

Coupled with the publicly-available CMUnited-98 source code [11], this article is designed to help researchers involved in the RoboCup software challenge [5] build upon our success. Throughout the article, we assume that the reader is familiar with the soccer server [1].

The rest of the article is organized as follows. Section 2 gives an overview of the agent architecture. Section 3 describes the agents' method of keeping an accurate and precise world model. Section 4 details the agents' low-level skills. Section 5 presents the CMUnited-98 collaborative coordination mechanisms. Section 6 summarizes the RoboCup-98 results and Section 7 concludes.

\section{Agent Architecture Overview}

CMUnited-98 agents are capable of perception, cognition, and action. By perceiving the world, they build a model of its current state. Then, based on a set of behaviors, they choose an action appropriate for the current world state.

\footnotetext{
* This research is sponsored in part by the DARPA/RL Knowledge Based Planning and Scheduling Initiative under grant number F30602-95-1-0018. The views and conclusions contained in this document are those of the authors and should not be interpreted as representing the official policies or endorsements, either expressed or implied, of the U. S. Government.
}

M. Asada and H. Kitano (Eds.): RoboCup-98, LNAI 1604, pp. 61-76, 1999.

(C) Springer-Verlag Heidelberg Berlin 1999 
A driving factor in the design of the agent architecture is the fact that the simulator operates in fixed cycles of length 100 msec. As presented in Section [1], the simulator accepts commands from clients throughout a cycle and then updates the world state all at once at the end of the cycle. Only one action command (dash, kick, turn, or catch) is executed for a given client during a given cycle.

Therefore, agents (simulator clients) should send exactly one action command to the simulator in every simulator cycle. If more than one command is sent in the same cycle, a random one is executed, possibly leading to undesired behavior. If no command is sent during a simulator cycle, an action opportunity has been lost: opponent agents who have acted during that cycle may gain an advantage.

In addition, since the simulator updates the world at the end of every cycle, it is advantageous to try to determine the state of the world at the end of the previous cycle when choosing an action for the current cycle. As such, the basic agent loop during a given cycle $t$ is as follows:

- Assume the agent has consistent information about the state of the world at the end of cycle $t-2$ and has sent an action during cycle $t-1$.

- While the server is still in cycle $t-1$, upon receipt of a sensation (see, hear, or sense_body), store the new information in temporary structures. Do not update the current state.

- When the server enters cycle $t$ (determined either by a running clock or by the receipt of a sensation with time stamp $t$ ), use all of the information available (temporary information from sensations and predicted effects of past actions) to update the world model to match the server's world state (the "real world state") at the end of cycle $t-1$. Then choose and send an action to the server for cycle $t$.

- Repeat for cycle $t+1$.

While the above algorithm defines the overall agent loop, much of the challenge is involved in updating the world model effectively and choosing an appropriate action. The remainder of this section goes into these processes in detail.

\section{World Modeling}

When acting based on a world model, it is important to have as accurate and precise a model of the world as possible at the time that an action is taken. In order to achieve this goal, CMUnited-98 agents gather sensory information over time, and process the information by incorporating it into the world model immediately prior to acting.

\subsection{Object Representation}

There are several objects in the world, such as the goals and the field markers which remain stationary and can be used for self-localization. Mobile objects are the agent itself, the ball, and 21 other players (10 teammates and 11 opponents). These objects are represented in a type hierarchy as illustrated in Figure 1.

Each agent's world model stores an instantiation of a stationary object for each goal, sideline, and field marker; a ball object for the ball; and 21 player 


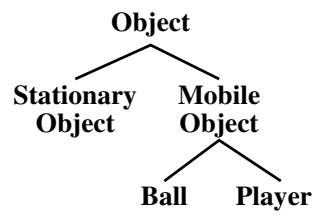

Fig. 1. The agent's object type hierarchy.

objects. Since players can be seen without their associated team and/or uniform number, the player objects are not identified with particular individual players. Instead, the variables for team and uniform number can be filled in as they become known.

Mobile objects are stored with confidence values within $[0,1]$ indicating the confidence with which their locations are known. The confidence values are needed because of the large amount of hidden state in the world: no object is seen consistently [2].

The variables associated with each object type are as follows:

\section{Object :}

- Global $(x, y)$ position coordinates

- Confidence within $[0,1]$ of the coordinates' accuracy

Stationary Object : nothing additional

Mobile Object :

- Global $(d x, d y)$ velocity coordinates

- Confidence within $[0,1]$ of the coordinates' accuracy

Ball : nothing additional

Player :

- Team

- Uniform number

- Global $\theta$ facing angle

- Confidence within [0,1] of the angle's accuracy

\subsection{Updating the World Model}

Information about the world can come from

- Visual information;

- Audial information;

- Sense_body information; and

- Predicted effects of previous actions.

Visual information arrives as relative distances and angles to objects in the player's view cone. Audial information could include information about global object locations from teammates. Sense_body information pertains to the client's own status including stamina, view mode, and speed. 
Whenever new information arrives, it is stored in temporary structures with time stamps and confidences (1 for visual information, possibly less for audial information). Visual information is stored as relative coordinates until the agent's exact location is determined.

When it is time to act during cycle $t$, all of the available information is used to best determine the server's world state at the end of cycle $t-1$. If no new information arrived pertaining to a given object, the velocity and actions taken are used by the predictor to predict the new position of the object and the confidence in that object's position and velocity are both decayed.

When the agent's world model is updated to match the end of simulator cycle $t-1$, first the agent's own position is updated to match the time of the last sight; then those of the ball and players are updated.

The Agent Itself: The following process is used to update the information about the agent:

- If new visual information has arrived:

- The agent's position can be determined accurately by using the relative coordinates of one seen line and the closest stationary object.

- If no visual information has arrived:

- Bring the velocity up to date, possibly incorporating the predicted effects of any actions (a dash) taken during the previous cycle.

- Using the previous position and velocity, predict the new position and velocity.

- If available, reset the agent's speed as per the sense_body information. Assume velocity is in the direction the agent is facing.

- Bring the player's stamina up to date either via the sense_body information or from the predicted action effects.

The Ball: The ball information is updated as follows:

- If there was new visual information, use the agent's absolute position at the time (determined above), and the ball's temporarily stored relative position to determine the ball's absolute position at the time of the sight.

- If velocity information is given as well, update the velocity. Otherwise, check if the old velocity is correct by comparing the new ball position with the expected ball position.

- If no new visual information arrived or the visual information was from cycle $t-1$, estimate its position and velocity for cycle $t$ using the values from cycle $t-1$. If the agent kicked the ball on the previous cycle, the predicted resulting ball motion is also taken into account.

- If the ball should be in sight (i.e. its predicted position is in the player's view cone), but isn't (i.e. visual information arrived, but no ball information was included), set the confidence to 0 .

- Information about the ball may have also arrived via communication from teammates. If any heard information would increase the confidence in the ball's position or velocity at this time, then it should be used as the correct information. Confidence in teammate information can be determined by the time of the information (did the teammate see the ball 
more recently?) and the teammate's distance to the ball (since players closer to the ball see it more precisely).

Ball velocity is particularly important for agents when determining whether or not (or how) to try to intercept the ball, and when kicking the ball. However, velocity information is often not given as part of the visual information string, especially when the ball is near the agent and kickable. Therefore, when necessary, the agents attempt to infer the ball's velocity indirectly from the current and previous ball positions.

Teammates and Opponents: In general, player positions and velocities are determined and maintained in the same way as in the case of the ball. A minor addition is that the direction a player is facing is also available from the visual information.

When a player is seen without full information about its identity, previous player positions can be used to help disambiguate the identity. Knowing the maximum distance a player can move in any given cycle, it is possible for the agent to determine whether a seen player could be the same as a previously identified player. If it is physically possible, the agent assumes that they are indeed the same player.

Since different players can see different regions of the field in detail, communication can play an important role in maintaining accurate information about player locations.

\section{Agent Skills}

Once the agent has determined the server's world state for cycle $t$ as accurately as possible, it can choose and send an action to be executed at the end of the cycle. In so doing, it must choose its local goal within the team's overall strategy. It can then choose from among several low-level skills which provide it with basic capabilities. The output of the skills are primitive movement commands.

The skills available to CMUnited-98 players include kicking, dribbling, ball interception, goaltending, defending, and clearing. The implementation details of these skills are described in this section.

The common thread among these skills is that they are all predictive, locally optimal skills (PLOS). They take into account predicted world models as well as predicted effects of future actions in order to determine the optimal primitive action from a local perspective, both in time and in space.

One simple example of PLOS is each individual agent's stamina management. The server models stamina as having a replenishable and a non-replenishable component. Each is only decremented when the current stamina goes below a fixed threshold. Each player monitors its own stamina level to make sure that it never uses up any of the non-replenishable component of its stamina. No matter how fast it should move according to the behavior the player is executing, it slows down its movement to keep itself from getting too tired. While such behavior might not be optimal in the context of the team's goal, it is locally optimal considering the agent's current tired state. 
Even though the skills are predictive, the agent commits to only one action during each cycle. When the time comes to act again, the situation is completely reevaluated. If the world is close to the anticipated configuration, then the agent will act similarly to the way it predicted on previous cycles. However, if the world is significantly different, the agent will arrive at a new sequence of actions rather than being committed to a previous plan. Again, it will only execute the first step in the new sequence.

\section{$4.1 \quad$ Kicking}

There are three points about the kick model of the server that should be understood before looking at our kicking style. First, a kick changes the ball's velocity by vector addition. That is, a kick accelerates the ball in a given direction, as opposed to setting the velocity. Second, an agent can kick the ball when it is in the "kickable area" which is a circle centered on the player (see Figure 2). Third, the ball and the player can collide. The server models a collision when the ball and player are overlapping at the end of a cycle. If there is a collision, the two bodies are separated and their velocities multiplied by -0.1 .

As a first level of abstraction when dealing with the ball, all reasoning is done as a desired trajectory for the ball for the next cycle. Before a kick is actually sent to the server, the difference between the ball's current velocity and the ball's desired velocity is used to determine the kick to actually perform. If the exact trajectory can not be obtained, the ball is kicked such that the direction is correct, even if the speed is not.

In order to effectively control the ball, a player must be able to kick the ball in any direction. In order to do so, the player must be able to move the ball from one side of its body to the other without the ball colliding with the player. This behavior is called the turnball behavior. It was developed based on code released by the PaSo'97 team[7]. The desired trajectory of a turnball kick is calculated by getting the ray from the ball's current position that is tangent to a circle around the player (see Figure 3 ).

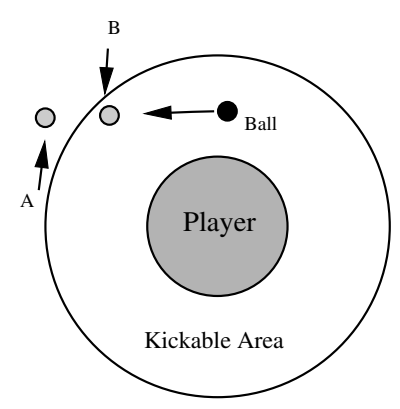

Fig. 2. Basic kicking with velocity prediction.

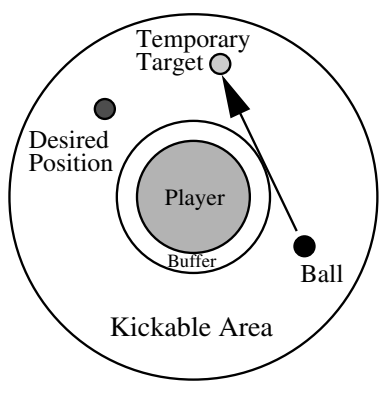

Fig. 3. The turnball skill. 
The next important skill is the ability to kick the ball in a given direction, either for passing or shooting. The first step is to figure out the target speed of the ball. If the agent is shooting, the target speed is the maximum ball speed, but for a pass, it might be better to kick the ball slower so that the receiving agent can intercept the ball more easily.

In order to get the ball to the desired speed, several kicks in succession are usually required. By putting the ball to the side of the player (relative to the desired direction of the kick) the agent can kick the ball several times in succession. If a higher ball speed is desired, the agent can use the turnball kicks to back the ball up so that enough kicks can be performed to accelerate the ball.

This skill is very predictive in that it looks at future velocities of the ball given slightly different possible kicks. In some cases, doing a weaker kick one cycle may keep the ball in the kickable area so that another kick can be executed the following cycle. In Figure 2, the agent must choose between two possible kicks. Kicking the ball to position A will result in the ball not being kickable next cycle; if the ball is already moving quickly enough, this action may be correct. However, a kick to position $B$ followed by a kick during the next cycle may result in a higher overall speed. Short term velocity prediction is the key to these decisions.

\subsection{Dribbling}

Dribbling is the skill which allows the player to move down the field while keeping the ball close to the player the entire time. The basic idea is fairly simple: alternate kicks and dashes so that after one of each, the ball is still close to the player.

Every cycle, the agent looks to see that if it dashes this cycle, the ball will be in its kickable area (and not be a collision) at the next cycle. If so, then the agent dashes, otherwise it kicks. A kick is always performed assuming that on the next cycle, the agent will dash. As an argument, the low-level dribbling code takes the angle relative to the direction of travel at which the player should aim the ball (see Figure 4). This is called the "dribble angle" and its valid values are $[-90,90]$. Deciding what the dribble angle should be is discussed in Section 4.3.

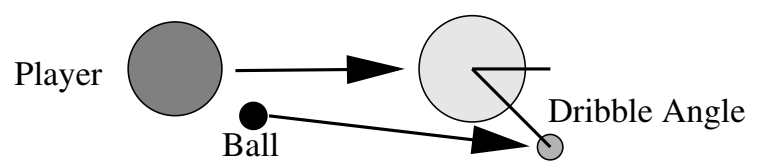

Current Position $\square$ Predicted Position in 2 cycles

Fig. 4. The basic dribbling skill.

First the predicted position of the agent (in 2 cycles) is calculated:

$$
p_{\text {new }}=p_{\text {current }}+v+(v * \text { pdecay }+a)
$$


where $p_{n e w}$ is the predicted player position, $p_{\text {current }}$ is the current position of the player, $v$ is the current velocity of the player, pdecay is the server parameter player_decay, and $a$ is the acceleration that a dash gives. The $a$ value is usually just the dash power times the dash_power_rate in the direction the player is facing, but stamina may need to be taken into account.

Added to $p_{n e w}$ is a vector in the direction of the dribble angle and length such that the ball is in the kickable area. This is the target position $p_{\text {target }}$ of the ball. Then the agent gets the desired ball trajectory by the following formula:

$$
\text { traj }=\frac{p_{\text {target }}-p_{\text {ball }}}{1+\text { bdecay }}
$$

where traj is the target trajectory of the ball, $p_{\text {ball }}$ is the current ball position, and bdecay is the server parameter ball_decay. This process is illustrated in Figure 4.

If for some reason this kick can not be done (it would be a collision for example), then a turnball kick is done to get the ball in the right position. Then the next cycle, a normal dribble kick should work.

As can be seen from these calculations, the basic dribbling is highly predictive of the positions and velocities of the ball and player. It is also quite local in that it only looks 2 cycles ahead and recomputes the best action every cycle.

\subsection{Smart Dribbling}

The basic dribbling takes one parameter that was mentioned above: the dribble angle. Smart dribbling is a skill layered on the basic dribbling skill that decides the best dribble angle based on opponent positions. Intuitively, the agent should keep the ball away from the opponents, so that if an opponent is on the left, the ball is kept on the right, and vice versa.

The agent considers all nearby opponents that it knows about. Each opponent is given a "vote" about what the dribble angle should be; each opponent votes for the valid angle $[-90,90]$ that is farthest from itself. For example, an opponent at 45 degrees, would vote for-90, while an opponent at -120 degrees would vote for 60 . Each opponent's vote is weighted by the distance and angle relative to the direction of motion. Closer opponents and opponents more in front of the agent are given more weight.

\subsection{Ball Interception}

There are two types of ball interception, referred to as active and passive interception. The passive interception is used only by the goaltender in some particular cases, while the rest of the team uses only the active interception. Each cycle, the interception target is recomputed so that the most up to date information about the world is used.

The active interception is similar to the one used by the Humboldt '97 team[3]. The active interception predicts the ball's position on successive cycles, and then tries to predict whether the player will be able to make it to that spot before the ball does, taking into account stamina and the direction that the player is facing. The agent aims for the earliest such spot. 
The passive interception is much more geometric. The agent determines the closest point along the ball's current trajectory that is within the field. By prediction based on the ball's velocity, the agent decides whether it can make it to that point before the ball. If so, then the agent runs towards that point.

\subsection{Goaltending}

The assumption behind the movement of the goaltender is that the worst thing that could happen to the goaltender is to lose sight of the ball. The sooner the goaltender sees a shot coming, the greater chance it has of preventing a goal. Therefore, the goaltender generally uses the widest view mode and uses backwards dashing when appropriate to keep the ball in view to position itself in situations that are not time-critical.

Every cycle that the ball is in the defensive zone, the goaltender looks to see if the ball is in the midst of a shot. It does this by extending the ray of the ball's position and velocity and intersecting that with the baseline of the field. If the intersection point is in the goaltender box and the ball has sufficient velocity to get there, the ball is considered to be a shot (though special care is used if an opponent can kick the ball this cycle). Using the passive interception if possible (see Section 4.4), the goaltender tries to get in the path of the ball and then run at the ball to grab it. This way, if the goaltender misses a catch or kick, the ball may still collide with the goaltender and thus be stopped.

When there is no shot coming the goaltender positions itself in anticipation of a future shot. Based on the angle of the ball relative to the goal, the goaltender picks a spot in the goal to guard; call this the "guard point." The further the ball is to the side of the field, the further the goaltender guards to that side. Then, a rectangle is computed that shrinks as the ball gets closer (though it never shrinks smaller than the goaltender box). The line from the guard point to the ball's current position is intersected with the rectangle, and that is the desired position of the goaltender.

\subsection{Defending}

CMUnited-98 agents are equipped with two different defending modes: opponent tracking and opponent marking. In both cases, a particular opponent player is selected as the target against which to defend. This opponent can either be selected individually or as a defensive unit via communication (the latter is the case in CMUnited-98).

In either case, the agent defends against this player by observing its position over time and position itself strategically so as to minimize its usefulness to the other team. When tracking, the agent stays between the opponent and the goal at a generous distance, thus blocking potential shots. When marking, the agent stays close to the opponent on the ball-opponent-goal angle bisector, making it difficult for the opponent to receive passes and shoot towards the goal. Defensive marking and tracking positions are illustrated in Figure 5.

When marking and tracking, it is important for the agent to have accurate knowledge about the positions of both the ball and the opponent (although the ball position isn't strictly relevant for tracking, it is used for the decision of 


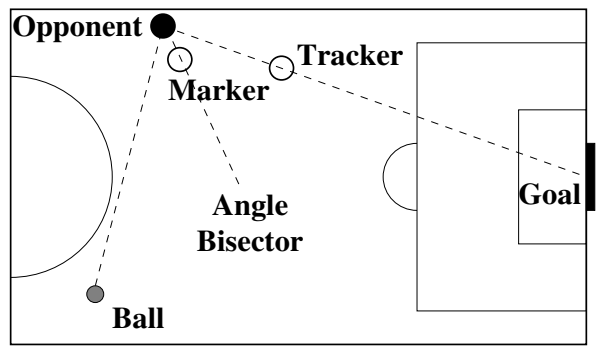

Fig. 5. Positioning for defensive tracking and marking.

whether or not to be tracking). Thus, when in the correct defensive position, the agent always turns to look at the object (opponent or ball) in which it is least confident of the correct position. The complete algorithm, which results in the behavior of doggedly following a particular opponent and glancing back and forth between the opponent and ball, is as follows:

- If the ball position is unknown, look for the ball.

- Else, if the opponent position is unknown, look for the opponent.

- Else, if not in the correct defensive position, move to that position.

- else, look towards the object, ball or opponent, which has been seen less recently (lower confidence value).

This defensive behavior is locally optimal in that it defends according to the opponent's current position, following it around rather than predicting its future location. However, in both cases, the defensive positioning is chosen in anticipation of the opponent's future possible actions, i.e. receiving a pass or shooting.

\subsection{Clearing}

Often in a defensive position, it is advantageous to just send the ball upfield, clearing it from the defensive zone. If the agent decides that it cannot pass or dribble while in a defensive situation, it will clear the ball. The important decision in clearing the ball is where to clear it to. The best clears are upfield, but not to the middle of the field (you don't want to center the ball for the opponents), and also away from the opponents.

The actual calculation is as follows. Every angle is evaluated with respect to its usefulness, and the expected degree of success. The usefulness is a sine curve with a maximum of 1 at 30 degrees, .5 at 90 degrees, and 0 at -90 , where a negative angle is towards the middle of the field. The actual equation is $(\Theta$ is in degrees):

$$
\text { usefulness }(\Theta)=\frac{\sin \left(\frac{3}{2} \Theta+45\right)+1}{2}
$$

The expected degree of success is evaluated by looking at an isosceles triangle with one vertex where the ball is, and congruent sides extending in the direction 
of the target being evaluated. For each opponent in the triangle, its distance from the center line of the triangle is divided by the distance from the player on that line. For opponent $\mathrm{C}$ in Figure 6 , these values are $w$ and $d$ respectively. The expected success is the product of all these quotients. In Figure 6, opponent A would not affect the calculation, being outside the triangle, while opponent $\mathrm{B}$ would lower the expected success to 0 , since it is on the potential clear line $(w=0)$.

By multiplying the usefulness and expected success together for each possible clear angle, and taking the maximum, the agent gets a crude approximation to maximizing the expected utility of a clear.

\section{Coordination}

Given all of the individual skills available to the CMUnited-98 clients, it becomes a significant challenge to coordinate the team so that the players are not all trying to do the same thing at the same time. Of course one and only one agent should execute the goaltending behavior. But it is not so clear how to determine when an agent should move towards the ball, when it should defend, when it should dribble, or clear, etc.

If all players act individually — constantly chase the ball and try to kick towards the opponent goal - they will all get tired, there will be nowhere to pass, and the opponents will have free reign over most of the field. Building upon the innovations of the CMUnited-97 simulator team [8], the CMUnited-98 team uses several complex coordination mechanisms, including reactive behavior modes, pre-compiled multi-agent plans and strategies, a flexible teamwork structure, a novel anticipatory offensive positioning scheme, and a sophisticated communication paradigm.

\subsection{Behavior Modes}

A player's top-level behavior decision is its behavior mode. Implemented as a rule-based system, the behavior mode determines the abstract behavior that the player should execute. For example, there is a behavior mode for the set of states in which the agent can kick the ball. Then, the decision of what to do with the ball is made by way of a more involved decision mechanism. On each action cycle, the first thing a player does is re-evaluate its behavior mode.

The behavior modes include:

Goaltend: Only used by the goaltender.

Localize: Find own field location if it's unknown.

Face Ball: Find the ball and look at it.

Handle Ball: Used when the ball is kickable.

Active Offense: Go to the ball as quickly as possible. Used when no teammate could get there more quickly.

Auxiliary Offense: Get open for a pass. Used when a nearby teammate has the ball.

Passive Offense: Move to a position likely to be useful offensively in the future. 
Active Defense: Go to the ball even though another teammate is already going. Used in the defensive end of the field.

Auxiliary Defense: Mark an opponent.

Passive Defense: Track an opponent or go to a position likely to be useful defensively in the future.

The detailed conditions and effects of each behavior mode are beyond the scope of this article. However, they will become more clear in subsequent sections as the role-based flexible team structure is described in Section 5.3.

\subsection{Locker-Room Agreement}

At the core of the CMUnited-98 coordination mechanism is what we call the Locker-Room Agreement [10]. Based on the premise that agents can periodically meet in safe, full-communication environments, the locker-room agreement specifies how they should act when in low-communication, time-critical, adversarial environments.

The locker-room agreement includes specifications of the flexible teamwork structure (Section 5.3) and the inter-agent communication paradigm (Section 5.5). A good example of the use of the locker-room agreement is CMUnited-98's ability to execute pre-compiled multi-agent plans after dead-ball situations. While it is often difficult to clear the ball from the defensive zone after goal kicks, CMUnited-98 players move to pre-specified locations and execute a series of passes that successfully move the ball out of their half of the field. Such "set plays" exist in the locker-room agreement for all dead-ball situations.

\subsection{Roles and Formations}

Like CMUnited-97, CMUnited-98 is organized around the concept of flexible formations consisting of flexible roles [10]. Each role specifies the behavior of the agent filling the role, both in terms of positioning on the field and in terms of the behavior modes that should be considered.

A formation is a collection of roles, again defined independently from the agents. The entire team can dynamically switch formations. CMUnited-98 used a standard formation with 4 defenders, 3 midfielders, and 3 forwards (4-3-3) at the beginnings of the games. If losing by enough goals relative to the time left in the game (as determined by the locker-room agreement), the team would switch to an offensive $3-3-4$ formation. When winning by enough, the team switched to a defensive 5-3-2 formation.

\subsection{SPAR}

The flexible roles defined in the CMUnited-97 software were an improvement over the concept of rigid roles. Rather than associating fixed $(x, y)$ coordinates with each position, an agent filling a particular role was given a range of coordinates in which it could position itself. Based on the ball's position on the field, the agent would position itself so as to increase the likelihood of being useful to the team in the future.

However, by taking into account the positions of other agents as well as that of the ball, an even more informed positioning decision can be made. The 
idea of strategic position by attraction and repulsion (SPAR) is one of the novel contributions of the CMUnited-98 research which has been applied to both the simulator and the small robot teams [12].

When positioning itself using SPAR, the agent uses a multi-objective function with attraction and repulsion points subject to several constraints. To formalize this concept, we introduce the following variables:

- $P$ - the desired position for the passive agent in anticipation of a passing need of its active teammate;

- $n$ - the number of agents on each team;

- $O_{i}$ - the current position of each opponent, $i=1, \ldots, n$;

- $T_{i}$ - the current position of each teammate, $i=1, \ldots,(n-1)$;

- $B$ - the current position of the active teammate and ball;

- $G$ - the position of the opponent's goal.

SPAR extends similar approaches of using potential fields for highly dynamic, multi-agent domains [6]. The probability of collaboration in the robotic soccer domain is directly related to how "open" a position is to allow for a successful pass. Thus, SPAR maximizes the distance from other robots and minimizes the distance to the ball and to the goal, namely:

- Repulsion from opponents, i.e., maximize the distance to each opponent: $\forall i, \max \operatorname{dist}\left(P, O_{i}\right)$

- Repulsion from teammates, i.e., maximize the distance to other passive teammates: $\forall i, \max \operatorname{dist}\left(P, T_{i}\right)$

- Attraction to the active teammate and ball: min $\operatorname{dist}(P, B)$

- Attraction to the opponent's goal: $\min \operatorname{dist}(P, G)$

This formulation is a multiple-objective function. To solve this optimization problem, we restate the problem as a single-objective function. As each term may have a different relevance (e.g. staying close to the goal may be more important than staying away from opponents), we want to apply a different weighting function to each term, namely $f_{O_{i}}, f_{T_{i}}, f_{B}$, and $f_{G}$, for opponents, teammates, the ball, and the goal, respectively. Our anticipation algorithm then maximizes a weighted single-objective function with respect to $P$ :

$\max \left(\sum_{i=1}^{n} f_{O_{i}}\left(\operatorname{dist}\left(P, O_{i}\right)\right)+\sum_{i=1}^{n-1} f_{T_{i}}\left(\operatorname{dist}\left(P, T_{i}\right)\right)-f_{B}(\operatorname{dist}(P, B))-f_{G}(\operatorname{dist}(P, G))\right)$

In our case, we use $f_{O_{i}}=f_{T_{i}}=x, f_{B}=0$, and $f_{G}=x^{2}$. For example, the last term of the objective function above expands to $(\operatorname{dist}(P, G))^{2}$.

One constraint in the simulator team relates to the position, or role, that the passive agent is playing relative to the position of the ball. The agent only considers locations that within one of the four rectangles, illustrated in Figure 7 : the one closest to the position home of the position that it is currently playing. This constraint helps ensure that the player with the ball will have several different passing options in different parts of the field. In addition, players don't need to consider moving too far from their positions to support the ball. 


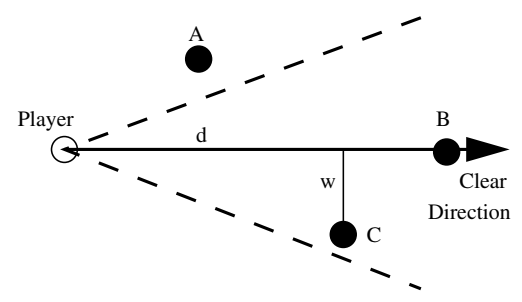

Fig. 6. Measuring the expected success of a clear.

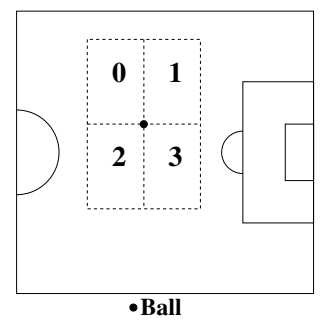

Fig. 7. The four possible rectangles, each with one corner at the ball's location, considered for positioning by simulator agents when using SPAR.

Since this position-based constraint already encourages players to stay near the ball, we set the ball-attraction weighting function $f_{B}$ to the constant function $y=0$. In addition to this first constraint, the agents observe three additional constraints. In total, the constraints in the simulator team are:

- Stay in an area near home position;

- Stay within the field boundaries;

- Avoid being in an offsides position;

- Stay in a position in which it would be possible to receive a pass.

This last constraint is evaluated by checking that there are no opponents in a cone with vertex at the ball and extending to the point in consideration.

In our implementation, the maximum of the objective function is estimated by sampling its values over a fine-grained mesh of points that satisfy the above constraints.

Using this SPAR algorithm, agents are able to anticipate the collaborative needs of their teammates by positioning themselves in such a way that the player with the ball would have several useful passing options.

\subsection{Communication}

The soccer server provides a challenging communication environment for teams of agents. With a single, low-bandwidth, unreliable communication channel for all 22 agents and limited communication range and capacity, agents must not rely on any particular message reaching any particular teammate. Nonetheless, when a message does get through, it can help distribute information about the state of the world as well as helping to facilitate team coordination. We identify a general communication environment for single-channel, low-bandwidth, unreliable communication environments [10].

All CMUnited-98 messages include a certain amount of state information from the speaker's perspective. Information regarding object position and teammate roles are all given along with the confidence values associated with this data. All teammates hearing the message can then use the information to augment their visual state information. 


\subsection{Ball Handling}

One of the most important decisions in the robotic soccer domain arises when the agent has control of the ball. In this state, it has the options of dribbling the ball in any direction, passing to any teammate, shooting the ball, clearing the ball, or simply controlling the ball.

In CMUnited-98, the agent uses a complex heuristic decision mechanism, incorporating a machine learning module, to choose its action. The best teammate to receive a potential pass (called potential receiver below) is determined by a decision tree trained off-line [9].

\section{Results}

In order to test individual components of the CMUnited-98 team, it is best to compile performance results for the team with and without these components as we have done elsewhere [10]. However, competition against other, independentlycreated teams is useful for evaluating the system as a whole.

At the RoboCup-98 competition, CMUnited-98 won all 8 of its games by a combined score of $66-0$, finishing first place in a field of 34 teams.

From observing the games, it was apparent that the CMUnited-98 low-level skills were superior in the first 6 games: CMUnited-98 agents were able to dribble around opponents, had many scoring opportunities, and suffered few shots against.

However, in the last 2 games, the CMUnited-98 strategic formations, communication, and ball-handling routines were put more to the test as the Windmill Wanderers (3rd place) and AT-Humboldt'98 (2nd place) also had similar lowlevel capabilities. In these games, CMUnited-98's abilities to use set plays to clear the ball from its defensive zone, to get past the opponents' defenders, and to maintain a cohesive defensive unit became very apparent. In addition, the fine points of the dribbling and goaltending skills came into play, with the players occasionally able to dribble around opponents for shots and with the CMUnited-98 goaltender making a particularly important save against the Windmill Wanderers.

Throughout the tournament, the CMUnited-98 software demonstrated its power as a complete multi-agent architecture in a real-time, noisy, adversarial environment.

\section{Conclusion}

The success of CMUnited-98 at RoboCup-98 was due to several technical innovations ranging from predictive locally optimal skills (PLOS) to strategic positioning using attraction and repulsion (SPAR). Building on the innovations of CMUnited-97, including flexible formation, a novel communication paradigm, and machine learning modules, CMUnited-98 successfully combines low-level individual and high-level strategic, collaborative reasoning in a single multi-agent architecture. 
For a more thorough understanding of the implementation details involved, the reader is encouraged to study the algorithms described here in conjunction with the CMUnited-98 source code [11]. Other RoboCup researchers and multi-agent researchers in general should be able to benefit and build from the innovations represented therein.

\section{References}

1. David Andre, Emiel Corten, Klaus Dorer, Pascal Gugenberger, Marius Joldos, Johan Kummeneje, Paul Arthur Navratil, Itsuki Noda, Patrick Riley, Peter Stone, Romoichi Takahashi, and Travlex Yeap. Soccer server manual, version 4.0. Technical Report RoboCup-1998-001, RoboCup, 1998. At URL http://ci.etl.go.jp/ noda/soccer/server/Documents.html.

2. Mike Bowling, Peter Stone, and Manuela Veloso. Predictive memory for an inaccessible environment. In Proceedings of the IROS-96 Workshop on RoboCup, pages 28-34, Osaka, Japan, November 1996.

3. Hans-Diter Burkhard, Markus Hannebauer, and Jan Wendler. AT humboldt development, practice and theory. In Hiroaki Kitano, editor, RoboCup-97: Robot Soccer World Cup I, pages 357-372. Springer Verlag, Berlin, 1998.

4. Hiroaki Kitano, Yasuo Kuniyoshi, Itsuki Noda, Minoru Asada, Hitoshi Matsubara, and Eïchi Osawa. RoboCup: A challenge problem for AI. AI Magazine, 18(1):7385, Spring 1997.

5. Hiroaki Kitano, Milind Tambe, Peter Stone, Manuela Veloso, Silvia Coradeschi, Eiichi Osawa, Hitoshi Matsubara, Itsuki Noda, and Minoru Asada. The RoboCup synthetic agent challenge 97. In Proceedings of the Fifteenth International Joint Conference on Artificial Intelligence, pages 24-29, San Francisco, CA, 1997. Morgan Kaufmann.

6. Jean-Claude Latombe. Robot Motion Planning. Kluwer, 1991.

7. E. Pagello, F. Montesello, A. D'Angelo, and C. Ferrari. A reactive architecture for RoboCup competition. In Hiroaki Kitano, editor, RoboCup-97: Robot Soccer World Cup I, pages 434-442. Springer Verlag, Berlin, 1998.

8. Peter Stone and Manuela Veloso. The CMUnited-97 simulator team. In Hiroaki Kitano, editor, RoboCup-97: Robot Soccer World Cup I, pages 387-397. Springer Verlag, Berlin, 1998.

9. Peter Stone and Manuela Veloso. A layered approach to learning client behaviors in the RoboCup soccer server. Applied Artificial Intelligence, 12:165-188, 1998.

10. Peter Stone and Manuela Veloso. Task decomposition, dynamic role assignment, and low-bandwidth communication for real-time strategic teamwork. Artificial Intelligence, 1999. To appear.

11. Peter Stone, Manuela Veloso, and Patrick Riley. CMUnited-98 source code, 1998. Accessible from http://www.cs.cmu.edu/ ${ }^{\sim}$ pstone/RoboCup/CMUnited98sim.html.

12. Manuela Veloso, Michael Bowling, Sorin Achim, Kwun Han, and Peter Stone. The CMUnited-98 champion small robot team. In Minoru Asada and Hiroaki Kitano, editors, RoboCup-98: Robot Soccer World Cup II. Springer Verlag, Berlin, 1999. 